method of treating peripheral early stage (T1, T2) NSCLC. It is considered as a valid option for treatment when surgery is high risk or declined. Many clinicians now question whether SABR can be considered as a genuine alternative to surgery for peripheral stage I NSCLC.

One concern about any comparison with surgery is that those patients who receive SABR may miss out on adjuvant chemotherapy due to lack of pathological nodal staging that would be done post operatively.

Our aim was to determine what proportion of cases this might represent.

Methods We carried out a retrospective review of surgical resections for lung cancer between 2008 and 2011 at a teaching hospital in Yorkshire. We identified those patients who were staged preoperatively as T1 or T2 with no nodal or distant metastases.

We also identified a subgroup of patients with peripheral tumours no greater than $5 \mathrm{~cm}$ in diameter (i.e. those potentially suitable for SABR).

We recorded post operative staging and whether they received adjuvant chemotherapy.

Results We identified 162 cases in total (81 female) of which $13.7 \%$ had nodal involvement on post operative staging. Of these, $72.7 \%$ received adjuvant chemotherapy. Within the subgroup with peripheral tumours $<5 \mathrm{~cm}$ there were 116 cases of which $7.0 \%$ had nodal involvement. Of these, $62.5 \%$ received adjuvant chemotherapy.

Conclusions Our findings show that a significant number of patients who are preoperatively staged as N0 do have hilar and/or mediastinal nodal involvement at the time of surgery. However, many of these patients have central tumours not suitable for SABR In the subgroup of patients with small peripheral tumours, that would potentially be suitable for SABR, this proportion is smaller.

It is thought that on direct comparison with surgery those who receive SABR might be more likely to develop recurrent disease due to nodal involvement outside of the radiotherapy field in some patients. Our results suggest that this proportion is small. Any effect on overall survival would be weighed against peri-operative morbidity and mortality.

\section{S95 SOCCAR: INTERNATIONALLY RESONANT RESULTS FROM A RANDOMISED TRIAL BASED ON UK CLINICAL PRACTISE}

doi:10.1136/thoraxjnl-2012-202678.101

1J Maguire, ${ }^{2} \mathrm{~N}$ O'Rourke, ${ }^{3} \mathrm{R}$ McNememin, ${ }^{4} \mathrm{C}$ Peedell, ${ }^{5} \mathrm{M}$ Snee, ${ }^{1} \mathrm{~V}$ Kelly, ${ }^{6}$ Khan. 'Liverpool Heart and Chest Hospital, Liverpool, UK; ${ }^{2}$ The Beatson West of Scotland Cancer Centre, Glasgow, UK; ${ }^{3}$ Northern Centre for Cancer Care, Newcastle, UK; ${ }^{4} \mathrm{James}$ Cook University Hospital, Middlesborough, UK; ${ }^{5}$ St James University Hospital, Leeds, UK, ${ }^{6}$ UCL Cancer Trials Centre, London, UK

Concurrent chemoradiation is now recognised as a standard of care for patients with inoperable Stage III NSCLC and good Performance Status. Two year survival rates in published trials ranges from $29 \%$ to $43 \%$, with median survival in the range of 14 to 17 months. An ongoing multi-centre study performed in the United States has recently reported median survival of 21 months for this group of patients, with no advantage from dose escalation from 60 to 74 Gy in conventional fractionation. In SOCCAR, we employed a standard UK radical lung cancer radiotherapy treatment schedule; 130 patients were randomised to receive 55Gy in 20 fractions over 4 weeks, given either concurrently or sequentially with cisplatinum and vinorelbine. Trial entry criteria were: pathologically confirmed stage III NSCLC, judged in-operable by a thoracic surgeon and treatable within a radical radiotherapy volume with V20 $<30 \%$ and $<12 \mathrm{~cm}$ oesophagus in PTV. Patients were required to have PS 0 or $1, \mathrm{FEV} 1>/=1 \mathrm{~L}$ and $\mathrm{TLCO}>/=$ to $50 \%$. Patients with weight loss and N3 disease were included, with no upper age limit. Characteristics were well balanced between the two arms.
Results Median survival is 27.4 months in the concurrent arm and 18.6 months in the sequential arm. Two year survival rates are $54 \%$ and $42 \%$ respectively. Toxicity was acceptable and similar in both arms. Documented local relapse was 10\% in the concurrent arm and $22 \%$ in the sequential arm. Quality of Life was measured prospectivly in all patients, there being no significant difference in either arm. In comparison with 16 published RCTs, comprising 1733 patients treated with concurrent chemoradiation, the concurrent treatment arm in SOCCAR ranks top in survival with comparable toxicity. These results are recognised as being of major relevance internationally and are the basis of a proposed multi-centre international study.

\section{Clinical studies in interstitial lung disease}

\section{S96 A COMBINATION SCORE OF RAISED SACE, LYMPHOPENIA AND HYPERGAMMIAGLOBULINAEMIA CORRELATES WITH ACTIVE DISEASE ON THORACIC CT SCAN OF PATIENTS WITH PULMONARY SARCOIDOSIS}

doi:10.1136/thoraxjnl-2012-202678.433

${ }^{1} Y R$ Kendrick, ${ }^{2}$ EJ Helm, ${ }^{2}$ R Benamore, 'LP Ho. ${ }^{1}$ Oxford Centre for Respiratory Medicine, Churchill Hospital and Weatherall Institute of Molecular Medicine, Oxford, UK; ${ }^{2}$ Thoracic Imaging Department, Churchill Hospital, Oxford, UK

Introduction Clinical decisions about treatment in pulmonary sarcoidosis, and phenotyping research studies are hampered by inability to objectively measure disease activity. We reviewed 120 consecutive patients from our Sarcoidosis Clinic and observed that raised SACE, immunoglobulin and/or presence of lymphopenia were associated with clinical decisions to change treatment. We question if these markers could be combined to form an objective measure of disease activity in pulmonary sarcoidosis.

Methods As there is no 'gold standard' for disease activity in sarcoidosis, we used thoracic CT scan to reflect disease activity on the basis that ground glass opacity, nodularity, consolidation, interlobular septal thickening and conglomeration reflect $T$ cell alveolit is, cellular infiltrate and granulomatous deposits ${ }^{1-2}$. Using Fleischner Society definition of terms ${ }^{3}$, we designed a scoring system ("CT activity score; CTAS") to quantify this, and examined if a composite clinical score "clinical activity score; CAS"; IgG $>13 \mathrm{~g} / \mathrm{l}=1$, Lymphocytes $<1 \times 10^{\wedge} 9 / 1=1$, sACE $<55 \mathrm{U} / 1=0,56-100 \mathrm{U} / 1=1$, $>100 \mathrm{U} / \mathrm{l}=2$ ) correlated with changes in CTAS. An enhanced CAS incorporating extent of defined CXR abnormalities was also examined. We collected data from 100 consecutive patients diagnosed according to WASOG/ATS criteria (with histological confirmation), who had thoracic CT scan, CXR and blood parameters within three months of each other. Two radiologists scored the CT scans blindly. We present results for the first 20 cases.

Results CTAS score (maximum possible 81 ) in our patients ranged from 0 to 40; CAS from 0-4 (maximum 5); when including CXR score - from 1-12 (maximum 12). We found a strong correlation between CAS and CTAS (Figure 1). No correlation was observed between the CTAS and FVC, TLCO or KCO, supporting the premise that severe irreversible functional loss can be inactive.

Conclusions A combination score incorporating lymphopenia, sACE levels, and hypergammaglobulinemia is strongly correlated with CT quantification of disease activity in pulmonary sarcoidosis. Addition of CXR scoring improved the correlation. This score could be used as an indicator of disease activity to aid clinical decisions on treatment, and paves the way for collation of larger numbers and longitudinal studies to further validate the tool.

\section{References}

1. Nishimura K et al. Radiology 1993.

2. Oberstein A et al. Sarc Vasc Diffuse Lung Dis 1997.

3. Hansell DM et al. Radiology 2008. 